\title{
Identification of unique immunogenic gluten peptides in Brazilian wheat flours using high resolution UPLC-MS
}

\author{
Thais de Oliveira Alves1-2; Carolina Thomaz dos Santos D'Almeida1-2; Gustavo Henrique
} Martins Ferreira de Souza3; Luiz Claudio Cameron2-4; Mariana Simões Larraz Ferreira1-2

(thais.o.alves@outlook.com, mariana.ferreira@unirio.br)

1-Lab. of Bioactives, Food and Nutrition Graduate Program (PPGAN); Federal University of State of Rio de Janeiro (UNIRIO). 2-Lab. of Protein Biochemistry, Center of Innovation in Mass Spectrometry (IMasS-LBP), UNIRIO. 3-MS Applications Research and Development Laboratory, Health Sciences Research, Waters Corporation, São Paulo, Brazil. 4 Department of Biochemistry and Sportomics, Olympic Laboratory, Brazil Olympic Committee, Rio de Janeiro, Brazil.

Gluten is a big protein network composed of monomers, formed by alcohol-soluble prolamins; and polymers, formed by insoluble glutelins, occurring in cereals endosperm, present in many cereal-based products, especially in those containing wheat. Celiac disease (CD) and other allergies are associated to gluten consumption and the treatment consists of the permanent exclusion of gluten from diet. For this reason, is extremely important to identify gluten proteins even in small concentrations. Proteomic approaches, such as LC-MS/MS, have been pointed as the most promising non-immunological techniques for gluten traces detection. This study aimed to identify immunogenic gluten peptides in different qualities of Brazilian wheat flour (Triticum aestivum) using UPLC-MS. Nine flours were classified and grouped based on gluten force as superior (SP) addressed to pasta making, medium (MD) used to bread making and low (LW) applied to biscuit and cake production. Gluten proteins were extracted and submitted to tryptic digestion. Peptides were submitted to NanoUPLC coupled to a Synapt G2-S (Waters Co.) mass spectrometer and data acquisition was performed using $\mathrm{MS}^{\mathrm{E}}$ method in triplicate. Data were processed on PLGS 3.0.1, peptides search was performed using a T. aestivum database from UniProt and ProPepper ${ }^{\mathrm{TM}}$ database to check their immunogenicity. Globally, 600 peptides were identified and after filtering around 150 were identified for each sample. About $14 \%$ of the identified peptides shown immunogenic action associated with prolamins. Amongst them, 10 peptides were common to the 3 samples and about 12 peptides were shared by two of them. Only 4 unique peptides were found in SP, 3 in MD and 5 in LW samples. The peptides were related to CD and other allergies, such as WDEIA (wheat-dependent exercise-induced anaphylaxis) allergy and atopic dermatitis. The immunogenicity of these peptides is known to be related to the presence of repetitive sequences of proline and glutamine. This study allowed the identification and the relative quantification of gluten peptides associated to immunologic diseases. Interestingly, flours classified as LW presented the highest abundance of epitopes, being 79\% more expressed in LW than SP and 90\% more than MD flours. Although the immunogenic activity is classically associated with gliadins, most of the immunogenic peptides belonged to glutenin subunits. In this work, LW flours showed greater potential to cause immunological reactions than the other qualities due to the higher number of epitopes in prolamins peptides. Keywords: celiac disease; prolamins; wheat quality. 